\title{
Fixed points for mappings satisfying some multi-valued contractions with $w$-distance
}

Zeqing Liu' ${ }^{1}$ Xiaochen Wang ${ }^{1}$, Shin Min Kang ${ }^{2 *}$ and Sun Young Cho ${ }^{3}$

"Correspondence:

smkang@gnu.ac.kr

${ }^{2}$ Department of Mathematics and

RINS, Gyeongsang National

University, Jinju, 660-701, Korea

Full list of author information is

available at the end of the article

\begin{abstract}
The existence of fixed points and iterative approximations for some nonlinear multi-valued contraction mappings in complete metric spaces with $w$-distance are proved. Two examples are included. The results presented in this paper extend, improve and unify many known results in recent literature.
\end{abstract}

MSC: $54 \mathrm{H} 25 ; 47 \mathrm{H} 10$

Keywords: multi-valued contractions; $w$-distance; fixed point theorems

\section{Introduction and preliminaries}

In 1996, Kada et al. [1] introduced the concept of $w$-distance and got some fixed point theorems for single-valued mappings under $w$-distance. In 2006, Feng and Liu [2, Theorem 3.1] proved the following fixed point theorem for a multi-valued contractive mapping, which generalizes the nice fixed point theorem due to Nadler [3, Theorem 5].

Theorem 1.1 ([2]) Let $(X, d)$ be a complete metric space and $T$ be a multi-valued mapping from $X$ into $\mathrm{CL}(X)$, where $\mathrm{CL}(X)$ is the family of all nonempty closed subsets of $X$. Assume that

$\left(\mathrm{c}_{1}\right)$ the mapping $f: X \rightarrow \mathbb{R}^{+}$, defined by $f(x)=d(x, T(x)), x \in X$, is lower semi-continuous;

$\left(c_{2}\right)$ there exist constants $b, c \in(0,1)$ with $c<b$ such that for any $x \in X$, there is $y \in T(x)$ satisfying

$$
b d(x, y) \leq f(x) \text { and } f(y) \leq c d(x, y) .
$$

Then $T$ has a fixed point in $X$.

In 2007, Klim and Wardowski [4, Theorem 2.1] extended Theorem 1.1 and proved the following result.

Theorem 1.2 ([4]) Let $(X, d)$ be a complete metric space and T be a multi-valued mapping from $X$ into $\mathrm{CL}(X)$ satisfying $\left(\mathrm{c}_{1}\right)$. Assume that

$\left(c_{3}\right)$ there exist $b \in(0,1)$ and $\varphi: \mathbb{R}^{+} \rightarrow[0, b)$ satisfying

$$
\limsup _{r \rightarrow t^{+}} \varphi(r)<b, \quad \forall t \in \mathbb{R}^{+},
$$

O2014 Liu et al.; licensee Springer. This is an Open Access article distributed under the terms of the Creative Commons Attribution License (http://creativecommons.org/licenses/by/2.0), which permits unrestricted use, distribution, and reproduction in any medium, provided the original work is properly cited. 
and for any $x \in X$, there is $y \in T(x)$ satisfying

$$
b d(x, y) \leq d(x, T(x)) \text { and } f(y) \leq \varphi(d(x, y)) d(x, y) .
$$

Then T has a fixed point in $X$.

In 2009 and 2010, Ćirić [5, Theorem 2.1] and Liu et al. [6, Theorems 2.1 and 2.3] established a few fixed point theorems for some multi-valued nonlinear contractions, which include the multi-valued contraction in Theorem 1.1 as a special case.

Theorem 1.3 ([5]) Let $(X, d)$ be a complete metric space and $T$ be a multi-valued mapping from $X$ into $\mathrm{CL}(X)$ satisfying $\left(\mathrm{c}_{1}\right)$. Assume that

$\left(\mathrm{c}_{4}\right)$ there exists a function $\varphi: \mathbb{R}^{+} \rightarrow[a, 1), 0<a<1$, satisfying

$$
\limsup _{r \rightarrow t^{+}} \varphi(r)<1, \quad \forall t \in \mathbb{R}^{+},
$$

and for any $x \in X$, there is $y \in T(x)$ satisfying

$$
\sqrt{\varphi(f(x))} d(x, y) \leq f(x) \text { and } f(y) \leq \varphi(f(x)) d(x, y) .
$$

Then $T$ has a fixed point in $X$.

Theorem 1.4 ([6]) Let $T$ be a multi-valued mapping from a complete metric space $(X, d)$ into $\mathrm{CL}(X)$ such that

$$
\begin{aligned}
& \text { for each } x \in X \text {, there exists } y \in T(x) \text { satisfying } \\
& \alpha(f(x)) d(x, y) \leq f(x) \text { and } f(y) \leq \beta(f(x)) d(x, y),
\end{aligned}
$$

where

$$
B= \begin{cases}{[0, \sup f(X)]} & \text { if } \sup f(X)<\infty, \\ {[0, \infty)} & \text { if } \sup f(X)=\infty,\end{cases}
$$

$\alpha: B \rightarrow(0,1]$ and $\beta: B \rightarrow[0,1)$ satisfy that

$$
\liminf _{r \rightarrow 0^{+}} \alpha(r)>0 \quad \text { and } \quad \limsup _{r \rightarrow t^{+}} \frac{\beta(r)}{\alpha(r)}<1, \quad \forall t \in[0, \sup f(X)) .
$$

Then

(a1) for each $x_{0} \in X$, there exist an orbit $\left\{x_{n}\right\}_{n \in \mathbb{N}_{0}}$ of $T$ and $z \in X$ such that $\lim _{n \rightarrow \infty} x_{n}=z$

(a2) $z$ is a fixed point of $T$ in $X$ if and only if the function $f(x)=d(x, T(x)), x \in X$, is $T$-orbitally lower semi-continuous at $z$.

Theorem 1.5 ([6]) Let $T$ be a multi-valued mapping from a complete metric space $(X, d)$ into $\mathrm{CL}(X)$ such that

$$
\begin{aligned}
& \text { for each } x \in X \text {, there exists } y \in T(x) \text { satisfying } \\
& \alpha(d(x, y)) d(x, y) \leq f(x) \text { and } f(y) \leq \beta(d(x, y)) d(x, y),
\end{aligned}
$$


where

$$
A= \begin{cases}{[0, \operatorname{diam}(X)]} & \text { if } \operatorname{diam}(X)<\infty \\ {[0, \infty)} & \text { if } \operatorname{diam}(X)=\infty\end{cases}
$$

$\alpha: A \rightarrow(0,1]$ and $\beta: A \rightarrow[0,1)$ satisfy that

$$
\liminf _{r \rightarrow t^{+}} \alpha(r)>0 \quad \text { and } \quad \limsup _{r \rightarrow t^{+}} \frac{\beta(r)}{\alpha(r)}<1, \quad \forall t \in[0, \operatorname{diam}(X)) \text {, }
$$

and one of $\alpha$ and $\beta$ is nondecreasing. Then

(a1) for each $x_{0} \in X$, there exist an orbit $\left\{x_{n}\right\}_{n \in \mathbb{N}_{0}}$ of $T$ and $z \in X$ such that $\lim _{n \rightarrow \infty} x_{n}=z$;

(a2) $z$ is a fixed point of $T$ in $X$ if and only if the function $f(x)=d(x, T(x)), x \in X$, is $T$-orbitally lower semi-continuous at $z$.

In 2011, Latif and Abdou [7, Theorem 2.1] generalized Theorem 1.3 and proved the following fixed point theorem for some multi-valued contractive mapping with $w$-distance.

Theorem 1.6 ([7]) Let $(X, d)$ be a complete metric space with a $w$-distance $w$, and let $T$ be a multi-valued mapping from $X$ into $\mathrm{CL}(X)$. Assume that

(c5) the mapping $f: X \rightarrow \mathbb{R}^{+}$, defined by $f_{w}(x)=w(x, T(x)), x \in X$, is lower semi-continuous;

$\left(\mathrm{c}_{6}\right)$ there exists a function $\varphi: \mathbb{R}^{+} \rightarrow[b, 1), 0<b<1$, satisfying

$$
\limsup _{r \rightarrow t^{+}} \varphi(r)<1, \quad \forall t \in \mathbb{R}^{+}
$$

and for any $x \in X$, there is $y \in T(x)$ satisfying

$$
\sqrt{\varphi\left(f_{w}(x)\right)} w(x, y) \leq f_{w}(x) \quad \text { and } \quad f_{w}(y) \leq \varphi\left(f_{w}(x)\right) w(x, y)
$$

Then there exists $v_{0} \in X$ such that $f_{w}\left(v_{0}\right)=0$. Further, if $w\left(v_{0}, v_{0}\right)=0$, then $v_{0} \in T\left(v_{0}\right)$.

The purpose of this paper is to prove the existence of fixed points and iterative approximations for some multi-valued contractive mappings with $w$-distance. Two examples with uncountably many points are included. The results presented in this paper extend, improve and unify Theorem 3.1 in [2], Theorem 2.1 in [4], Theorems 2.1 and 2.2 in [5], Theorems 2.1 and 2.3 in [6], Theorems 2.1-2.3 and 2.5 in [7], Theorem 6 in [8], Theorems 2.2 and 2.4 in [9] and Theorems 3.1-3.4 in [10].

Throughout this paper, we assume that $\mathbb{R}^{+}=[0, \infty), \mathbb{N}_{0}=\mathbb{N} \cup\{0\}$, where $\mathbb{N}$ denotes the set of all positive integers.

Definition 1.7 ([1]) A function $w: X \times X \rightarrow \mathbb{R}^{+}$is called a $w$-distance in $X$ if it satisfies the following:

$\left(\mathrm{w}_{1}\right) \quad w(x, z) \leq w(x, y)+w(y, z), \forall x, y, z \in X$ 
$\left(\mathrm{w}_{2}\right)$ for each $x \in X$, a mapping $w(x, \cdot): X \rightarrow \mathbb{R}^{+}$is lower semi-continuous, that is, if $\left\{y_{n}\right\}_{n \in \mathbb{N}}$ is a sequence in $X$ with $\lim _{n \rightarrow \infty} y_{n}=y \in X$, then $w(x, y) \leq \liminf _{n \rightarrow \infty} w\left(x, y_{n}\right)$;

$\left(\mathrm{w}_{3}\right)$ for any $\varepsilon>0$, there exists $\delta>0$ such that $w(z, x) \leq \delta$ and $w(z, y) \leq \delta$ imply $d(x, y) \leq \varepsilon$.

For any $u \in X, D \subseteq X, w$-distance $w$ and $T: X \rightarrow \mathrm{CL}(X)$, put

$$
\begin{aligned}
& d(u, D)=\inf _{y \in D} d(u, y), \quad w(u, D)=\inf _{y \in D} w(u, y), \\
& f(u)=d(u, T(u)), \quad f_{w}(u)=w(u, T(u)), \\
& \operatorname{diam}(X)=\sup \{d(x, y): x, y \in X\}, \quad \operatorname{diam}\left(X_{w}\right)=\sup \{w(x, y): x, y \in X\}, \\
& A_{w}= \begin{cases}{\left[0, \operatorname{diam}\left(X_{w}\right)\right]} & \text { if } \operatorname{diam}\left(X_{w}\right)<\infty, \\
{[0, \infty)} & \text { if } \operatorname{diam}\left(X_{w}\right)=\infty\end{cases}
\end{aligned}
$$

and

$$
B_{w}= \begin{cases}{\left[0, \sup f_{w}(X)\right]} & \text { if } \sup f_{w}(X)<\infty \\ {[0, \infty)} & \text { if } \sup f_{w}(X)=\infty\end{cases}
$$

A sequence $\left\{x_{n}\right\}_{n \in \mathbb{N}_{0}}$ in $X$ is called an orbit of $T$ at $x_{0} \in X$ if $x_{n} \in T\left(x_{n-1}\right)$ for all $n \in \mathbb{N}$. A function $g: X \rightarrow \mathbb{R}^{+}$is said to be T-orbitally lower semi-continuous at $z \in X$ if $g(z) \leq$ $\liminf _{n \rightarrow \infty} g\left(x_{n}\right)$ for each orbit $\left\{x_{n}\right\}_{n \in \mathbb{N}_{0}} \subset X$ of $T$ with $\lim _{n \rightarrow \infty} x_{n}=z$. A function $\varphi: A_{w} \rightarrow$ $\mathbb{R}^{+}$is called subadditive in $A_{w}$ if $\varphi(s+t) \leq \varphi(s)+\varphi(t)$ for all $s, t \in A_{w}$. A function $\varphi: A_{w} \rightarrow$ $\mathbb{R}^{+}$is called strictly inverse in $A_{w}$ if $\varphi(t)<\varphi(s)$ implies that $t<s$.

Lemma 1.8 ([11]) Let $(X, d)$ be a metric space with a $w$-distance $w$ and $D \in \mathrm{CL}(X)$. Suppose that there exists $u \in X$ such that $w(u, u)=0$. Then $w(u, D)=0$ if and only if $u \in D$.

\section{Fixed point theorems}

In this section we prove the existence of fixed points and iterative approximations for some nonlinear multi-valued contraction mappings in complete metric spaces with $w$-distance.

Theorem 2.1 Let $(X, d)$ be a complete metric space, $w$ be a $w$-distance in $X$ and $T$ be a multi-valued mapping from $X$ into $\mathrm{CL}(X)$ such that

$$
\begin{aligned}
& \text { for each } x \in X \text {, there exists } y \in T(x) \text { satisfying } \\
& \alpha\left(f_{w}(x)\right) \varphi(w(x, y)) \leq f_{w}(x) \quad \text { and } \quad f_{w}(y) \leq \beta\left(f_{w}(x)\right) \psi(w(x, y)),
\end{aligned}
$$

where

$\alpha$ and $\beta$ are functions from $B_{w}$ into $(0,1]$ and $[0,1)$, respectively, with

$\beta(0)<\alpha(0), \quad \liminf _{r \rightarrow 0^{+}} \alpha(r)>0 \quad$ and $\quad \limsup _{r \rightarrow t^{+}} \frac{\beta(r)}{\alpha(r)}<1, \quad \forall t \in B_{w}$,

$\varphi$ and $\psi$ are functions from $A_{w}$ into $\mathbb{R}^{+}$with $\psi(t) \leq \varphi(t), \quad \forall t \in A_{w}$ and

$\varphi$ is subadditive in $A_{w}$ and satisfies that either 
$\varphi$ is strictly inverse in $A_{w}, \varphi(0)=0, \varphi(t)>0, \quad \forall t \in A_{w} \backslash\{0\}$

or

$\varphi$ is strictly increasing in $A_{w}$ and $\lim _{t \rightarrow 0^{+}} \varphi^{-1}(t)=0$, where $\varphi^{-1}$

stands for the inverse function of $\varphi$.

Then

(a1) for each $x_{0} \in X$, there exists an orbit $\left\{x_{n}\right\}_{n \in \mathbb{N}_{0}}$ of $T$ such that $\lim _{n \rightarrow \infty} x_{n}=u_{0}$ for some $u_{0} \in X$

(a2) $f_{w}\left(u_{0}\right)=0$ if and only if the function $f_{w}$ is T-orbitally lower semi-continuous at $u_{0}$;

(a3) $u_{0} \in T\left(u_{0}\right)$ provided that $w\left(u_{0}, u_{0}\right)=0=f_{w}\left(u_{0}\right)$;

(a4) $T$ has a fixed point in $X$ if for each orbit $\left\{z_{n}\right\}_{n \in \mathbb{N}_{0}}$ of $T$ in $X$ and $v \in X$ with $v \notin T(v)$, one of the following conditions is satisfied:

$$
\begin{aligned}
& \inf \left\{w\left(z_{n}, v\right)+\varphi\left(w\left(z_{n}, z_{n+1}\right)\right): n \in \mathbb{N}_{0}\right\}>0 \\
& \inf \left\{w\left(z_{n}, v\right)+w\left(z_{n}, T\left(z_{n}\right)\right): n \in \mathbb{N}_{0}\right\}>0
\end{aligned}
$$

Proof Firstly, we prove (a1). Let

$$
\gamma(t)=\frac{\beta(t)}{\alpha(t)}, \quad \forall t \in B_{w} .
$$

It follows from (2.1) that for each $x_{0} \in X$, there exists $x_{1} \in T\left(x_{0}\right)$ satisfying

$$
\alpha\left(f_{w}\left(x_{0}\right)\right) \varphi\left(w\left(x_{0}, x_{1}\right)\right) \leq f_{w}\left(x_{0}\right) \quad \text { and } \quad f_{w}\left(x_{1}\right) \leq \beta\left(f_{w}\left(x_{0}\right)\right) \psi\left(w\left(x_{0}, x_{1}\right)\right)
$$

which together with (2.3) and (2.8) yields that

$$
\begin{aligned}
f_{w}\left(x_{1}\right) & \leq \beta\left(f_{w}\left(x_{0}\right)\right) \psi\left(w\left(x_{0}, x_{1}\right)\right) \leq \beta\left(f_{w}\left(x_{0}\right)\right) \varphi\left(w\left(x_{0}, x_{1}\right)\right) \\
& \leq \beta\left(f_{w}\left(x_{0}\right)\right) \frac{f_{w}\left(x_{0}\right)}{\alpha\left(f_{w}\left(x_{0}\right)\right)}=\gamma\left(f_{w}\left(x_{0}\right)\right) f_{w}\left(x_{0}\right) .
\end{aligned}
$$

Continuing this process, we choose easily an orbit $\left\{x_{n}\right\}_{n \in \mathbb{N}_{0}}$ of $T$ satisfying

$$
\begin{aligned}
& x_{n+1} \in T\left(x_{n}\right), \quad \alpha\left(f_{w}\left(x_{n}\right)\right) \varphi\left(w\left(x_{n}, x_{n+1}\right)\right) \leq f_{w}\left(x_{n}\right) \quad \text { and } \\
& f_{w}\left(x_{n+1}\right) \leq \beta\left(f_{w}\left(x_{n}\right)\right) \psi\left(w\left(x_{n}, x_{n+1}\right)\right), \quad \forall n \in \mathbb{N}_{0} .
\end{aligned}
$$

It follows from (2.3), (2.8) and (2.9) that

$$
\begin{aligned}
f_{w}\left(x_{n+1}\right) & \leq \beta\left(f_{w}\left(x_{n}\right)\right) \psi\left(w\left(x_{n}, x_{n+1}\right)\right) \leq \beta\left(f_{w}\left(x_{n}\right)\right) \varphi\left(w\left(x_{n}, x_{n+1}\right)\right) \\
& \leq \beta\left(f_{w}\left(x_{n}\right)\right) \frac{f_{w}\left(x_{n}\right)}{\alpha\left(f_{w}\left(x_{n}\right)\right)}=\gamma\left(f_{w}\left(x_{n}\right)\right) f_{w}\left(x_{n}\right), \quad \forall n \in \mathbb{N}_{0} .
\end{aligned}
$$

Now we claim that

$$
\lim _{n \rightarrow \infty} f_{w}\left(x_{n}\right)=0
$$


Notice that the ranges of $\alpha$ and $\beta,(2.2)$ and (2.8) ensure that

$$
0 \leq \gamma(t)<1, \quad \forall t \in B_{w}
$$

Using (2.10) and (2.12), we conclude that $\left\{f_{w}\left(x_{n}\right)\right\}_{n \in \mathbb{N}_{0}}$ is a nonnegative and nonincreasing sequence, which means that there is a constant $a \geq 0$ satisfying

$$
\lim _{n \rightarrow \infty} f_{w}\left(x_{n}\right)=a .
$$

Suppose that $a>0$. Using (2.2), (2.8), (2.10), (2.12) and (2.13), we obtain that

$$
\begin{aligned}
a & =\limsup _{n \rightarrow \infty} f_{w}\left(x_{n+1}\right) \leq \limsup _{n \rightarrow \infty}\left[\gamma\left(f_{w}\left(x_{n}\right)\right) f_{w}\left(x_{n}\right)\right] \\
& \leq \limsup _{n \rightarrow \infty} \gamma\left(f_{w}\left(x_{n}\right)\right) \limsup _{n \rightarrow \infty} f_{w}\left(x_{n}\right) \\
& \leq a \limsup _{r \rightarrow a^{+}} \gamma(r)<a,
\end{aligned}
$$

which is a contradiction. Thus $a=0$, that is, (2.11) holds.

Next we claim that $\left\{x_{n}\right\}_{n \in \mathbb{N}_{0}}$ is a Cauchy sequence. Put

$$
b=\limsup _{n \rightarrow \infty} \gamma\left(f_{w}\left(x_{n}\right)\right) \text { and } c=\liminf _{n \rightarrow \infty} \alpha\left(f_{w}\left(x_{n}\right)\right) .
$$

It follows from (2.2), (2.8), (2.12) and (2.14) that

$$
0 \leq b<1 \quad \text { and } \quad c>0 .
$$

Let $p \in(0, c)$ and $q \in(b, 1)$. Because of (2.14) and (2.15), we deduce that there exists some $n_{0} \in \mathbb{N}$ such that

$$
\gamma\left(f_{w}\left(x_{n}\right)\right)<q \quad \text { and } \quad \alpha\left(f_{w}\left(x_{n}\right)\right)>p, \quad \forall n \geq n_{0},
$$

which together with (2.9) and (2.10) yields that

$$
f_{w}\left(x_{n+1}\right) \leq q f_{w}\left(x_{n}\right) \quad \text { and } \quad \varphi\left(w\left(x_{n}, x_{n+1}\right)\right) \leq \frac{f_{w}\left(x_{n}\right)}{p}, \quad \forall n \geq n_{0}
$$

which implies that

$$
f_{w}\left(x_{n+1}\right) \leq q^{n+1-n_{0}} f_{w}\left(x_{n_{0}}\right) \quad \text { and } \quad \varphi\left(w\left(x_{n}, x_{n+1}\right)\right) \leq \frac{f_{w}\left(x_{n_{0}}\right)}{p} q^{n-n_{0}}, \quad \forall n \geq n_{0}
$$

By means of $\left(w_{1}\right),(2.3)$ and (2.16), we deduce that

$$
\begin{aligned}
\varphi\left(w\left(x_{n}, x_{m}\right)\right) & \leq \sum_{k=n}^{m-1} \varphi\left(w\left(x_{k}, x_{k+1}\right)\right) \leq \sum_{k=n}^{m-1} \frac{f_{w}\left(x_{n_{0}}\right)}{p} q^{k-n_{0}} \\
& \leq \frac{f_{w}\left(x_{n_{0}}\right)}{p(1-q)} q^{n-n_{0}}, \quad \forall m>n \geq n_{0} .
\end{aligned}
$$


Given $\varepsilon>0$, denote by $\delta$ the constant in $\left(\mathrm{w}_{3}\right)$ corresponding to $\varepsilon$. Assume that (2.4) holds. It follows from $\varphi(\delta)>0$ and $q \in(b, 1)$ that there exists a positive integer $N \geq n_{0}$ satisfying

$$
\frac{f_{w}\left(x_{n_{0}}\right)}{p(1-q)} q^{n-n_{0}}<\varphi(\delta), \quad \forall n \geq N
$$

Combining (2.17) and (2.18), we infer that

$$
\max \left\{\varphi\left(w\left(x_{N}, x_{m}\right)\right), \varphi\left(w\left(x_{N}, x_{n}\right)\right)\right\} \leq \frac{f_{w}\left(x_{n_{0}}\right)}{p(1-q)} q^{n-n_{0}}<\varphi(\delta), \quad \forall m>n \geq N
$$

which together with (2.4) guarantees that

$$
\max \left\{w\left(x_{N}, x_{m}\right), w\left(x_{N}, x_{n}\right)\right\}<\delta, \quad \forall m>n>N
$$

It follows from $\left(\mathrm{w}_{3}\right)$ and (2.19) that

$$
d\left(x_{m}, x_{n}\right) \leq \varepsilon, \quad \forall m>n>N .
$$

It is clear that (2.20) yields that $\left\{x_{n}\right\}_{n \in \mathbb{N}_{0}}$ is a Cauchy sequence.

Assume that (2.5) holds. Since $\varphi$ is strictly increasing, so does $\varphi^{-1}$. It follows from (2.5) and $q \in(b, 1)$ that there exists a positive integer $N \geq n_{0}$ satisfying

$$
\varphi^{-1}\left(\frac{f_{w}\left(x_{n_{0}}\right)}{p(1-q)} q^{n-n_{0}}\right)<\delta, \quad \forall n \geq N
$$

which together with (2.5) and (2.17) means that

$$
w\left(x_{n}, x_{m}\right)=\varphi^{-1}\left(\varphi\left(w\left(x_{n}, x_{m}\right)\right)\right) \leq \varphi^{-1}\left(\frac{f_{w}\left(x_{n_{0}}\right)}{p(1-q)} q^{n-n_{0}}\right)<\delta, \quad \forall m>n \geq N
$$

which ensures that (2.19) and (2.20) hold. Consequently, $\left\{x_{n}\right\}_{n \in \mathbb{N}_{0}}$ is a Cauchy sequence.

It follows from completeness of $(X, d)$ that there is some $u_{0} \in X$ such that $\lim _{n \rightarrow \infty} x_{n}=u_{0}$.

Secondly, we prove (a2). Suppose that $f_{w}$ is $T$-orbitally lower semi-continuous at $u_{0}$. Let $\left\{x_{n}\right\}_{n \in \mathbb{N}_{0}}$ be the orbit of $T$ defined by (2.9) and satisfy (2.11). It follows from (2.11) that

$$
0 \leq w\left(u_{0}, T\left(u_{0}\right)\right)=f_{w}\left(u_{0}\right) \leq \liminf _{n \rightarrow \infty} f_{w}\left(x_{n}\right)=0,
$$

which means that $f_{w}\left(u_{0}\right)=0$. Conversely, suppose that $f_{w}\left(u_{0}\right)=0$ for some $u_{0} \in X$. Let $\left\{y_{n}\right\}_{n \in \mathbb{N}_{0}}$ be an arbitrary orbit of $T$ in $X$ with $\lim _{n \rightarrow \infty} y_{n}=u_{0}$. It follows that

$$
f_{w}\left(u_{0}\right)=0 \leq \liminf _{n \rightarrow \infty} f_{w}\left(y_{n}\right)
$$

that is, $f_{w}$ is $T$-orbitally lower semi-continuous at $u_{0}$.

Thirdly, we prove (a3). Note that $T\left(u_{0}\right)$ is closed and

$$
w\left(u_{0}, u_{0}\right)=0=f_{w}\left(u_{0}\right)=w\left(u_{0}, T\left(u_{0}\right)\right) .
$$

It follows from Lemma 1.8 that $u_{0} \in T\left(u_{0}\right)$. 
Finally, we prove (a4). Assume that $\left\{x_{n}\right\}_{n \in \mathbb{N}_{0}}$ is the orbit of $T$ defined by (2.9) and that it satisfies (2.11), (2.16), (2.17) and $\lim _{n \rightarrow \infty} x_{n}=u_{0} \in X$. Clearly, (2.16) and $q \in(b, 1)$ mean that

$$
\lim _{n \rightarrow \infty} \varphi\left(w\left(x_{n}, x_{n+1}\right)\right)=0 .
$$

Now we claim that

$$
\lim _{n \rightarrow \infty} w\left(x_{n}, u_{0}\right)=0
$$

In order to prove (2.22), we consider two possible cases as follows.

Case 1. Assume that (2.4) holds. Let $\varepsilon>0$ be given. Notice that $\varphi(\varepsilon)>0$ and $q \in(b, 1)$. It follows that there exists a positive integer $N>n_{0}$ satisfying

$$
\frac{f_{w}\left(x_{n_{0}}\right)}{p(1-q)} q^{n-n_{0}}<\varphi(\varepsilon), \quad \forall n \geq N
$$

which together with (2.17) yields that

$$
\varphi\left(w\left(x_{n}, x_{m}\right)\right) \leq \frac{f_{w}\left(x_{n_{0}}\right)}{p(1-q)} q^{n-n_{0}}<\varphi(\varepsilon), \quad \forall m>n \geq N .
$$

Since $\varphi$ is strictly inverse, it follows that

$$
w\left(x_{n}, x_{m}\right)<\varepsilon, \quad \forall m>n \geq N .
$$

Letting $m \rightarrow \infty$ in the above inequality and using $\left(\mathrm{w}_{2}\right)$, we get that

$$
w\left(x_{n}, u_{0}\right) \leq \liminf _{m \rightarrow \infty} w\left(x_{n}, x_{m}\right) \leq \varepsilon, \quad \forall n \geq N
$$

that is, (2.22) holds.

Case 2. Assume that (2.5) holds. It follows from (2.5) and (2.17) that

$$
w\left(x_{n}, x_{m}\right)=\varphi^{-1}\left(\varphi\left(w\left(x_{n}, x_{m}\right)\right)\right) \leq \varphi^{-1}\left(\frac{f_{w}\left(x_{n_{0}}\right)}{p(1-q)} q^{n-n_{0}}\right), \quad \forall m>n \geq n_{0},
$$

which together with $\left(\mathrm{w}_{2}\right)$ and $(2.5)$ ensures that

$$
w\left(x_{n}, u_{0}\right) \leq \liminf _{m \rightarrow \infty} w\left(x_{n}, x_{m}\right) \leq \varphi^{-1}\left(\frac{f_{w}\left(x_{n_{0}}\right)}{p(1-q)} q^{n-n_{0}}\right) \rightarrow 0 \quad \text { as } n \rightarrow \infty,
$$

that is, (2.22) holds.

Suppose that $u_{0} \notin T\left(u_{0}\right)$. Let $v=u_{0}$ and $z_{n}=x_{n}$ for each $n \in \mathbb{N}_{0}$. Assume that (2.6) holds. Making use of (2.6), (2.21) and (2.22), we conclude that

$$
0<\inf \left\{w\left(x_{n}, u_{0}\right)+\varphi\left(w\left(x_{n}, x_{n+1}\right)\right): n \in \mathbb{N}_{0}\right\}=0,
$$

which is a contradiction. Assume that (2.7) holds. By virtue of (2.7), (2.11) and (2.22), we infer that

$$
0<\inf \left\{w\left(x_{n}, u_{0}\right)+w\left(x_{n}, x_{n+1}\right): n \in \mathbb{N}_{0}\right\}=0,
$$

which is also a contradiction. Consequently, $u_{0} \in T\left(u_{0}\right)$. This completes the proof. 
Theorem 2.2 Let $(X, d)$ be a complete metric space, $w$ be a $w$-distance in $X$ and $T$ be a multi-valued mapping from $X$ into $\mathrm{CL}(X)$ such that (2.3) and one of (2.4) and (2.5) hold and

$$
\begin{aligned}
& \text { for each } x \in X \text {, there exists } y \in T(x) \text { satisfying } \\
& \alpha(w(x, y)) \varphi(w(x, y)) \leq f_{w}(x) \text { and } f_{w}(y) \leq \beta(w(x, y)) \psi(w(x, y)),
\end{aligned}
$$

where

$\alpha$ and $\beta$ are functions from $A_{w}$ into $(0,1]$ and $[0,1)$, respectively, with

$$
\beta(0)<\alpha(0), \quad \liminf _{r \rightarrow 0^{+}} \alpha(r)>0 \quad \text { and } \quad \limsup _{r \rightarrow t^{+}} \frac{\beta(r)}{\alpha(r)}<1, \quad \forall t \in A_{w}
$$

and

one of $\alpha$ and $\beta$ is nondecreasing in $A_{w}$.

Then (a1)-(a4) hold.

Proof Firstly, we prove (a1). Let

$$
\gamma(t)=\frac{\beta(t)}{\alpha(t)}, \quad \forall t \in A_{w} .
$$

Notice that the ranges of $\alpha$ and $\beta,(2.24)$ and (2.26) ensure that

$$
0 \leq \gamma(t)<1, \quad \forall t \in A_{w} .
$$

It follows from (2.23) that for each $x_{0} \in X$, there exists $x_{1} \in T\left(x_{0}\right)$ satisfying

$$
\alpha\left(w\left(x_{0}, x_{1}\right)\right) \varphi\left(w\left(x_{0}, x_{1}\right)\right) \leq f_{w}\left(x_{0}\right) \quad \text { and } \quad f_{w}\left(x_{1}\right) \leq \beta\left(w\left(x_{0}, x_{1}\right)\right) \psi\left(w\left(x_{0}, x_{1}\right)\right) \text {, }
$$

which together with (2.3) and (2.26) means that

$$
\begin{aligned}
f_{w}\left(x_{1}\right) & \leq \beta\left(w\left(x_{0}, x_{1}\right)\right) \psi\left(w\left(x_{0}, x_{1}\right)\right) \leq \beta\left(w\left(x_{0}, x_{1}\right)\right) \varphi\left(w\left(x_{0}, x_{1}\right)\right) \\
& \leq \beta\left(w\left(x_{0}, x_{1}\right)\right) \frac{f_{w}\left(x_{0}\right)}{\alpha\left(w\left(x_{0}, x_{1}\right)\right)}=\gamma\left(w\left(x_{0}, x_{1}\right)\right) f_{w}\left(x_{0}\right) .
\end{aligned}
$$

Continuing this process, we choose easily an orbit $\left\{x_{n}\right\}_{n \in \mathbb{N}_{0}}$ of $T$ satisfying

$$
\begin{aligned}
& x_{n+1} \in T\left(x_{n}\right), \quad \alpha\left(w\left(x_{n}, x_{n+1}\right)\right) \varphi\left(w\left(x_{n}, x_{n+1}\right)\right) \leq f_{w}\left(x_{n}\right) \quad \text { and } \\
& f_{w}\left(x_{n+1}\right) \leq \beta\left(w\left(x_{n}, x_{n+1}\right)\right) \psi\left(w\left(x_{n}, x_{n+1}\right)\right), \quad \forall n \in \mathbb{N}_{0},
\end{aligned}
$$

which together with (2.3) and (2.26) gives that

$$
\begin{aligned}
f_{w}\left(x_{n+1}\right) & \leq \beta\left(w\left(x_{n}, x_{n+1}\right)\right) \psi\left(w\left(x_{n}, x_{n+1}\right)\right) \leq \beta\left(w\left(x_{n}, x_{n+1}\right)\right) \varphi\left(w\left(x_{n}, x_{n+1}\right)\right) \\
& \leq \beta\left(w\left(x_{n}, x_{n+1}\right)\right) \frac{f_{w}\left(x_{n}\right)}{\alpha\left(w\left(x_{n}, x_{n+1}\right)\right)}=\gamma\left(w\left(x_{n}, x_{n+1}\right)\right) f_{w}\left(x_{n}\right), \quad \forall n \in \mathbb{N}_{0}
\end{aligned}
$$


and

$$
\begin{aligned}
\varphi\left(w\left(x_{n+1}, x_{n+2}\right)\right) & \leq \frac{f_{w}\left(x_{n+1}\right)}{\alpha\left(w\left(x_{n+1}, x_{n+2}\right)\right)} \\
& \leq \frac{\beta\left(w\left(x_{n}, x_{n+1}\right)\right)}{\alpha\left(w\left(x_{n+1}, x_{n+2}\right)\right)} \psi\left(w\left(x_{n}, x_{n+1}\right)\right), \quad \forall n \in \mathbb{N}_{0} .
\end{aligned}
$$

Now we claim that

$$
w\left(x_{n+1}, x_{n+2}\right) \leq w\left(x_{n}, x_{n+1}\right), \quad \forall n \in \mathbb{N}_{0} .
$$

Suppose that there exists $n_{0} \in \mathbb{N}_{0}$ satisfying

$$
w\left(x_{n_{0}+1}, x_{n_{0}+2}\right)>w\left(x_{n_{0}}, x_{n_{0}+1}\right) .
$$

Let (2.4) hold. It follows from (2.3), (2.25), (2.26), (2.30) and (2.32) that

$$
\begin{aligned}
\varphi\left(w\left(x_{n_{0}+1}, x_{n_{0}+2}\right)\right) & \leq \frac{\beta\left(w\left(x_{n_{0}}, x_{n_{0}+1}\right)\right)}{\alpha\left(w\left(x_{n_{0}+1}, x_{n_{0}+2}\right)\right)} \psi\left(w\left(x_{n_{0}}, x_{n_{0}+1}\right)\right) \\
& \leq \max \left\{\gamma\left(w\left(x_{n_{0}}, x_{n_{0}+1}\right)\right), \gamma\left(w\left(x_{n_{0}+1}, x_{n_{0}+2}\right)\right)\right\} \varphi\left(w\left(x_{n_{0}}, x_{n_{0}+1}\right)\right) .
\end{aligned}
$$

If $\varphi\left(w\left(x_{n_{0}}, x_{n_{0}+1}\right)\right)=0$, it follows from $(2.33)$ that $\varphi\left(w\left(x_{n_{0}+1}, x_{n_{0}+2}\right)\right)=0$. Thus (2.4) and (2.32) guarantee that

$$
0 \leq w\left(x_{n_{0}}, x_{n_{0}+1}\right)<w\left(x_{n_{0}+1}, x_{n_{0}+2}\right)=0,
$$

which is a contradiction; if $\varphi\left(w\left(x_{n_{0}}, x_{n_{0}+1}\right)\right)>0,(2.4),(2.26),(2.27)$ and (2.33) yield that

$$
\begin{aligned}
\varphi\left(w\left(x_{n_{0}+1}, x_{n_{0}+2}\right)\right) & \leq \max \left\{\gamma\left(w\left(x_{n_{0}}, x_{n_{0}+1}\right)\right), \gamma\left(w\left(x_{n_{0}+1}, x_{n_{0}+2}\right)\right)\right\} \varphi\left(w\left(x_{n_{0}}, x_{n_{0}+1}\right)\right) \\
& <\varphi\left(w\left(x_{n_{0}}, x_{n_{0}+1}\right)\right) .
\end{aligned}
$$

Since $\varphi$ is strictly inverse, it follows from (2.32) and (2.34) that

$$
w\left(x_{n_{0}+1}, x_{n_{0}+2}\right)<w\left(x_{n_{0}}, x_{n_{0}+1}\right)<w\left(x_{n_{0}+1}, x_{n_{0}+2}\right),
$$

which is impossible.

Let (2.5) hold. Notice that $\varphi$ is strictly increasing. It follows from (2.3), (2.26), (2.27), (2.30) and (2.32) that

$$
\begin{aligned}
\varphi\left(w\left(x_{n_{0}+1}, x_{n_{0}+2}\right)\right) & \leq \frac{\beta\left(w\left(x_{n_{0}}, x_{n_{0}+1}\right)\right)}{\alpha\left(w\left(x_{n_{0}+1}, x_{n_{0}+2}\right)\right)} \psi\left(w\left(x_{n_{0}}, x_{n_{0}+1}\right)\right) \\
& \leq \max \left\{\gamma\left(w\left(x_{n_{0}}, x_{n_{0}+1}\right)\right), \gamma\left(w\left(x_{n_{0}+1}, x_{n_{0}+2}\right)\right)\right\} \varphi\left(w\left(x_{n_{0}}, x_{n_{0}+1}\right)\right) \\
& \leq \varphi\left(w\left(x_{n_{0}}, x_{n_{0}+1}\right)\right) \\
& <\varphi\left(w\left(x_{n_{0}+1}, x_{n_{0}+2}\right)\right)
\end{aligned}
$$


which is absurd. Hence (2.31) holds. That is, $\left\{w\left(x_{n}, x_{n+1}\right)\right\}_{n \in \mathbb{N}_{0}}$ is a nonincreasing and nonnegative sequence. It follows that $\lim _{n \rightarrow \infty} w\left(x_{n}, x_{n+1}\right)=d$ for some $d \geq 0$.

Now we claim that (2.11) holds. Using (2.27) and (2.29), we conclude that $\left\{f_{w}\left(x_{n}\right)\right\}_{n \in \mathbb{N}_{0}}$ is a nonnegative and nonincreasing sequence. Consequently, (2.13) is satisfied for some $a \geq 0$. Suppose that $a>0$. Using (2.13), (2.24), (2.27) and (2.29), we obtain that

$$
\begin{aligned}
a & =\limsup _{n \rightarrow \infty} f_{w}\left(x_{n+1}\right) \leq \limsup _{n \rightarrow \infty}\left[\gamma\left(w\left(x_{n}, x_{n+1}\right)\right) f_{w}\left(x_{n}\right)\right] \\
& \leq \limsup _{n \rightarrow \infty} \gamma\left(w\left(x_{n}, x_{n+1}\right)\right) \limsup _{n \rightarrow \infty} f_{w}\left(x_{n}\right) \leq a \limsup _{t \rightarrow d^{+}} \gamma(t) \\
& <a,
\end{aligned}
$$

which is a contradiction. Thus $a=0$, that is, (2.11) holds.

Next we claim that $\left\{x_{n}\right\}_{n \in \mathbb{N}_{0}}$ is a Cauchy sequence. Put

$$
b=\limsup _{n \rightarrow \infty} \gamma\left(w\left(x_{n}, x_{n+1}\right)\right) \quad \text { and } \quad c=\liminf _{n \rightarrow \infty} \alpha\left(w\left(x_{n}, x_{n+1}\right)\right) .
$$

It follows from (2.24), (2.27), (2.29) and (2.35) that (2.15) holds. Let $p \in(0, c)$ and $q \in(b, 1)$. Because of (2.15) and (2.35), we deduce that there exists some $n_{0} \in \mathbb{N}$ such that

$$
\gamma\left(w\left(x_{n}, x_{n+1}\right)\right)<q \quad \text { and } \quad \alpha\left(w\left(x_{n}, x_{n+1}\right)\right)>p, \quad \forall n \geq n_{0},
$$

which together with (2.28) and (2.29) yields that

$$
f_{w}\left(x_{n+1}\right) \leq q f_{w}\left(x_{n}\right) \quad \text { and } \quad \varphi\left(w\left(x_{n}, x_{n+1}\right)\right) \leq \frac{f_{w}\left(x_{n}\right)}{p}, \quad \forall n \geq n_{0} .
$$

The rest of the proof is similar to that of Theorem 2.1 and is omitted. This completes the proof.

\section{Remarks and illustrative examples}

In this section we construct two nontrivial examples to illustrate the results in Section 2.

Remark 3.1 Theorem 2.1 extends Theorem 3.1 in [2], Theorem 2.1 in [5], Theorem 2.1 in [6], Theorems 2.1 and 2.2 in [7], Theorems 2.2 and 2.4 in [9], and Theorems 3.1 and 3.2 in [10]. Example 3.2 below shows that Theorem 2.1 extends substantially Theorem 3.1 in [2] and Theorem 2.1 in [5] and differs from Theorems 5 and 6 in [8] and Theorem 2.1 in [4].

Example 3.2 Let $X=[0,1] \cup\left\{\frac{6}{5}\right\}$ be endowed with the Euclidean metric $d=|\cdot|$ and $u_{0}=0$. Define $w: X \times X \rightarrow \mathbb{R}^{+}, T: X \rightarrow \operatorname{CL}(X), \alpha:\left[0, \frac{1}{4}\right] \rightarrow(0,1], \beta:\left[0, \frac{1}{4}\right] \rightarrow[0,1)$ and $\varphi, \psi:$ $\left[0, \frac{6}{5}\right] \rightarrow \mathbb{R}^{+}$by

$$
\begin{aligned}
& w(x, y)=y, \quad \forall x, y \in X, \\
& T(x)= \begin{cases}\left\{\frac{x}{4}\right\}, & \forall x \in\left[0, \frac{2}{5}\right) \cup\left(\frac{2}{5}, 1\right], \\
\left\{\frac{1}{10}, \frac{1}{3}\right\}, & \forall x \in\left\{\frac{2}{5}, \frac{6}{5}\right\},\end{cases} \\
& \alpha(t)=\frac{8+t}{9}, \quad \beta(t)=\frac{2+t}{3}, \quad \forall t \in\left[0, \frac{1}{4}\right]
\end{aligned}
$$


and

$$
\varphi(t)=t, \quad \psi(t)=\min \{t,|1-t|\}, \quad \forall t \in\left[0, \frac{6}{5}\right]
$$

It is easy to see that $A_{w}=\left[0, \frac{6}{5}\right], B_{w}=\left[0, \frac{1}{4}\right],(2.3),(2.4)$ and $(2.5)$ hold and

$$
f_{w}(x)=w(x, T(x))= \begin{cases}\frac{x}{4}, & \forall x \in\left[0, \frac{2}{5}\right) \cup\left(\frac{2}{5}, 1\right] \\ \frac{1}{10}, & \forall x \in\left\{\frac{2}{5}, \frac{6}{5}\right\}\end{cases}
$$

is $T$-orbitally lower semi-continuous at $u_{0}$,

$$
\begin{aligned}
& \beta(0)=\frac{2}{3}<\frac{8}{9}=\alpha(0), \quad \liminf _{r \rightarrow 0^{+}} \alpha(r)=\frac{8}{9}>0, \\
& \limsup _{r \rightarrow t^{+}} \frac{\beta(r)}{\alpha(r)}=\limsup _{r \rightarrow t^{+}}\left(\frac{2+r}{3} \cdot \frac{9}{8+r}\right)=\frac{6+3 t}{8+t}<1, \quad \forall t \in\left[0, \frac{1}{4}\right] .
\end{aligned}
$$

For $x \in\left[0, \frac{2}{5}\right) \cup\left(\frac{2}{5}, 1\right]$, there exists $y=\frac{x}{4} \in T(x)=\left\{\frac{x}{4}\right\}$ satisfying

$$
\alpha\left(f_{w}(x)\right) \varphi(w(x, y))=\frac{8+\frac{x}{4}}{9} \cdot \frac{x}{4} \leq \frac{x}{4}=f_{w}(x)
$$

and

$$
f_{w}(y)=\frac{x}{16} \leq \frac{2+\frac{x}{4}}{3} \cdot \min \left\{\frac{x}{4}, 1-\frac{x}{4}\right\}=\beta\left(f_{w}(x)\right) \psi(w(x, y)) .
$$

For $x \in\left\{\frac{2}{5}, \frac{6}{5}\right\}$, there exists $y=\frac{1}{10} \in T(x)=\left\{\frac{1}{10}, \frac{1}{3}\right\}$ satisfying

$$
\alpha\left(f_{w}(x)\right) \varphi(w(x, y))=\frac{8+\frac{1}{10}}{9} \cdot \frac{1}{10} \leq \frac{1}{10}=f_{w}(x)
$$

and

$$
f_{w}(y)=\frac{1}{40} \leq \frac{2+\frac{1}{10}}{3} \cdot \min \left\{\frac{1}{10}, 1-\frac{1}{10}\right\}=\beta\left(f_{w}(x)\right) \psi(w(x, y))
$$

Put $v \in X \backslash\{0\}$ and $\left\{z_{n}\right\}_{n \in \mathbb{N}_{0}}$ is an orbit of $T$ in $X$. It is easy to verify that $\lim _{n \rightarrow \infty} z_{n}=u_{0}=0$ and

$$
\begin{aligned}
\inf & \left\{w\left(z_{n}, v\right)+\varphi\left(w\left(z_{n}, z_{n+1}\right)\right): n \in \mathbb{N}_{0}\right\} \\
\quad= & \inf \left\{v+z_{n+1}: n \in \mathbb{N}_{0}\right\} \\
\quad= & v+u_{0}=v>0 .
\end{aligned}
$$

Hence (2.1), (2.2) and (2.6) hold, that is, the conditions of Theorem 2.1 are fulfilled. Thus Theorem 2.1 guarantees that (a1)-(a4) hold. Moreover, $T$ has a fixed point $u_{0}=0 \in X$.

Now we show that Theorem 2.1 in [5] is unapplicable in proving the existence of fixed points for the multi-valued mapping $T$. Otherwise there exists a function $\varphi: \mathbb{R}^{+} \rightarrow[a, 1)$, 
$0<a<1$, such that

$$
\limsup _{r \rightarrow t^{+}} \varphi(r)<1, \quad \forall t \in \mathbb{R}^{+},
$$

and for any $x \in X$ there is $y \in T(x)$ satisfying

$$
\sqrt{\varphi(f(x))} d(x, y) \leq f(x)
$$

and

$$
f(y) \leq \varphi(f(x)) d(x, y)
$$

Note that

$$
f(x)=d(x, T(x))= \begin{cases}\frac{3}{4} x, & \forall x \in\left[0, \frac{2}{5}\right) \cup\left(\frac{2}{5}, 1\right] \\ \frac{1}{15}, & x=\frac{2}{5}, \\ \frac{13}{15}, & x=\frac{6}{5} .\end{cases}
$$

Put $x=\frac{2}{5}$. For $y \in T(x)=\left\{\frac{1}{10}, \frac{1}{3}\right\}$, we discuss two cases as follows.

Case 1. $y=\frac{1}{10}$. It follows from (3.2) and (3.3) that

$$
\frac{3}{10} \sqrt{\varphi\left(\frac{1}{15}\right)}=\sqrt{\varphi\left(f\left(\frac{2}{5}\right)\right)} d\left(\frac{2}{5}, \frac{1}{10}\right)=\sqrt{\varphi(f(x))} d(x, y) \leq f(x)=f\left(\frac{2}{5}\right)=\frac{1}{15}
$$

and

$$
\frac{3}{40}=f\left(\frac{1}{10}\right)=f(y) \leq \varphi(f(x)) d(x, y)=\varphi\left(f\left(\frac{2}{5}\right)\right) d\left(\frac{2}{5}, \frac{1}{10}\right)=\frac{3}{10} \varphi\left(\frac{1}{15}\right),
$$

which imply that

$$
0.25=\frac{1}{4} \leq \varphi\left(\frac{1}{15}\right) \leq \frac{4}{81}=0.049,
$$

which is impossible.

Case 2. $y=\frac{1}{3}$. It follows from (3.3) that

$$
\frac{1}{4}=f\left(\frac{1}{3}\right)=f(y) \leq \varphi(f(x)) d(x, y)=\varphi\left(f\left(\frac{2}{5}\right)\right) d\left(\frac{2}{5}, \frac{1}{3}\right)=\frac{1}{15} \varphi\left(\frac{1}{15}\right),
$$

which together with $\varphi\left(\mathbb{R}^{+}\right) \subseteq[a, 1)$ yields that

$$
\frac{15}{4} \leq \varphi\left(\frac{1}{15}\right)<1
$$

which is absurd. 
Next we show that Theorem 5 in [8] is useless in proving the existence of fixed points for the multi-valued mapping $T$. Otherwise there exists a function $\varphi: \mathbb{R}^{+} \rightarrow[0,1)$ such that (3.1) holds, and for any $x \in X$ there is $y \in T(x)$ satisfying

$$
d(x, y) \leq(2-\varphi(d(x, y))) f(x)
$$

and

$$
f(y) \leq \varphi(d(x, y)) d(x, y) .
$$

Put $x=\frac{2}{5}$. For $y \in T(x)=\left\{\frac{1}{10}, \frac{1}{3}\right\}$, we discuss two cases as follows.

Case 1. $y=\frac{1}{10}$. It follows from (3.4) that

$$
\frac{3}{10}=d\left(\frac{2}{5}, \frac{1}{10}\right)=d(x, y) \leq(2-\varphi(d(x, y))) f(x)=\left(2-\varphi\left(\frac{3}{10}\right)\right) \frac{1}{15},
$$

which together with $\varphi\left(\mathbb{R}^{+}\right) \subseteq[0,1)$ yields that

$$
0 \leq \varphi\left(\frac{3}{10}\right) \leq-\frac{5}{2}<0
$$

which is a contradiction.

Case 2. $y=\frac{1}{3}$. It follows from (3.4) that

$$
\frac{1}{4}=f\left(\frac{1}{3}\right)=f(y) \leq \varphi(d(x, y)) d(x, y)=\varphi\left(d\left(\frac{2}{5}, \frac{1}{3}\right)\right) d\left(\frac{2}{5}, \frac{1}{3}\right)=\frac{1}{15} \varphi\left(\frac{1}{15}\right)
$$

which together with $\varphi\left(\mathbb{R}^{+}\right) \subseteq[0,1)$ gives that

$$
\frac{15}{4} \leq \varphi\left(\frac{1}{15}\right)<1,
$$

which is impossible.

Finally we show that Theorem 6 in [8] is futile in proving the existence of fixed points for the multi-valued mapping $T$. Otherwise there exist functions $\varphi: \mathbb{R}^{+} \rightarrow(0,1), b: \mathbb{R}^{+} \rightarrow$ $[b, 1), b>0$ such that

$$
\varphi(t)<b(t), \quad \limsup _{r \rightarrow t^{+}} \varphi(r)<\limsup _{r \rightarrow t^{+}} b(r), \quad \forall t \in \mathbb{R}^{+},
$$

and for any $x \in X$, there is $y \in T(x)$ satisfying (3.5) and

$$
b(d(x, y)) d(x, y) \leq f(x) .
$$

Put $x=\frac{2}{5}$. For $y \in T(x)=\left\{\frac{1}{10}, \frac{1}{3}\right\}$, we discuss two cases as follows.

Case 1. $y=\frac{1}{10}$. It follows from (3.7) and (3.5) that

$$
\frac{3}{10} b\left(\frac{3}{10}\right)=b\left(d\left(\frac{2}{5}, \frac{1}{10}\right)\right) d\left(\frac{2}{5}, \frac{1}{10}\right)=b(d(x, y)) d(x, y) \leq f(x)=f\left(\frac{2}{5}\right)=\frac{1}{15}
$$


and

$$
\frac{3}{40}=f\left(\frac{1}{10}\right)=f(y) \leq \varphi(d(x, y)) d(x, y)=\frac{3}{10} \varphi\left(\frac{3}{10}\right)
$$

which together with (3.6) means that

$$
b\left(\frac{3}{10}\right) \leq \frac{2}{9}<\frac{1}{4} \leq \varphi\left(\frac{3}{10}\right)<b\left(\frac{3}{10}\right)
$$

which is absurd.

Case 2. $y=\frac{1}{3}$. It follows from (3.5) that

$$
\frac{1}{4}=f\left(\frac{1}{3}\right)=f(y) \leq \varphi(d(x, y)) d(x, y)=\varphi\left(d\left(\frac{2}{5}, \frac{1}{3}\right)\right) d\left(\frac{2}{5}, \frac{1}{3}\right)=\frac{1}{15} \varphi\left(\frac{1}{15}\right)
$$

which together with $\varphi\left(\mathbb{R}^{+}\right) \subseteq[0,1)$ gives that

$$
\frac{15}{4} \leq \varphi\left(\frac{1}{15}\right)<1
$$

which is impossible.

Observe that Theorem 6 in [8] extends Theorem 3.1 in [2], Theorem 2.1 in [4] and Theorem 2.2 in [5]. It follows that Theorem 3.1 in [2], Theorem 2.1 in [4] and Theorem 2.2 in [5] are not applicable in proving the existence of fixed points for the multi-valued mapping $T$.

Remark 3.3 Theorem 2.2 extends, improves and unifies Theorem 3.1 in [2], Theorem 2.1 in [4], Theorem 2.2 in [5], Theorem 2.3 in [6], Theorems 2.3 and 2.5 in [7], Theorem 6 in [8], and Theorems 3.3 and 3.4 in [10]. The following example reveals that Theorem 2.2 generalizes indeed the corresponding results in $[2,4,5,8]$.

Example 3.4 Let $X=[0, \infty)$ be endowed with the Euclidean metric $d=|\cdot|$ and $p \geq 1$ be a constant. Put $u_{0}=0$. Define $w: X \times X \rightarrow \mathbb{R}^{+}, T: X \rightarrow \mathrm{CL}(X), \alpha:[0, \infty) \rightarrow(0,1]$ and $\varphi, \psi:[0, \infty) \rightarrow \mathbb{R}^{+}$by $\beta:[0, \infty) \rightarrow[0,1)$ by

$$
\begin{aligned}
& w(x, y)=y^{p}, \quad \forall x, y \in X, \\
& T(x)= \begin{cases}{\left[\frac{x^{2}}{2}, \frac{x}{2}\right],} & \forall x \in[0,1), \\
{\left[\frac{1}{9}, \frac{1}{4}\right],} & \forall x \in[1, \infty),\end{cases} \\
& \alpha(t)=\frac{5+t^{\frac{1}{p}}}{10}, \quad \beta(t)=\frac{3+t^{\frac{1}{p}}}{10}, \quad \forall t \in[0, \infty)
\end{aligned}
$$

and

$$
\varphi(t)=t, \quad \forall t \in[0, \infty), \quad \psi(t)= \begin{cases}t, & \forall t \in[0,1), \\ \frac{1}{2}, & \forall t \in[1, \infty)\end{cases}
$$


It is easy to see that $A_{w}=[0, \infty),(2.3),(2.4)$ and (2.5) hold, $w$ is a $w$-distance in $X$ and

$$
f_{w}(x)=w(x, T(x))= \begin{cases}\left(\frac{x^{2}}{2}\right)^{p}, & \forall x \in[0,1), \\ \frac{1}{9^{p}}, & \forall x \in[1, \infty)\end{cases}
$$

is $T$-orbitally lower semi-continuous in $X, \alpha$ and $\beta$ are nondecreasing,

$$
\beta(0)=\frac{3}{10}<\frac{1}{2}=\alpha(0), \quad \liminf _{r \rightarrow 0^{+}} \alpha(r)=\frac{1}{2}>0
$$

and

$$
\limsup _{r \rightarrow t^{+}} \frac{\beta(r)}{\alpha(r)}=\frac{3+t^{\frac{1}{p}}}{5+t^{\frac{1}{p}}}<1, \quad \forall t \in A_{w} .
$$

Put $x \in[0,1)$ and $y=\frac{x^{2}}{2} \in T(x)$. Note that

$$
5+y \leq 10 \text { and }\left(\frac{y}{2}\right)^{p} \leq \frac{1}{4^{p}} \leq \frac{3+y}{10}
$$

imply that

$$
\alpha(w(x, y)) \varphi(w(x, y))=\frac{5+y}{10} \cdot y^{p} \leq y^{p}=f_{w}(x)
$$

and

$$
f_{w}(y)=\left(\frac{y^{2}}{2}\right)^{p} \leq \frac{3+y}{10} \cdot y^{p}=\beta(w(x, y)) \psi(w(x, y)) .
$$

Put $x \in[1, \infty)$ and $y=\frac{1}{9} \in T(x)=\left[\frac{1}{9}, \frac{1}{4}\right]$. It follows that

$$
\alpha(w(x, y)) \varphi(w(x, y))=\frac{5+\frac{1}{9}}{10} \cdot \frac{1}{9^{p}} \leq \frac{1}{9^{p}}=f_{w}(x)
$$

and

$$
f_{w}(y)=\frac{1}{182^{p}} \leq \frac{3+\frac{1}{9}}{10} \cdot \frac{1}{9^{p}}=\beta(w(x, y)) \psi(w(x, y)) .
$$

Let $v \in X \backslash\{0\}$ and $\left\{z_{n}\right\}_{n \in \mathbb{N}_{0}}$ be an orbit of $T$. It is easy to verify that $\lim _{n \rightarrow \infty} z_{n}=0$ and

$$
\begin{gathered}
\inf \left\{w\left(z_{n}, v\right)+\varphi\left(w\left(z_{n}, z_{n+1}\right)\right): n \in \mathbb{N}_{0}\right\} \\
\quad=\inf \left\{v^{p}+z_{n+1}^{p}: n \in \mathbb{N}_{0}\right\}=v^{p}>0 .
\end{gathered}
$$

That is, (2.6) and (2.23)-(2.25) hold. Thus the conditions of Theorem 2.2 are satisfied. Consequently, Theorem 2.2 ensures that (a1)-(a4) hold and $u_{0}=0$ is a fixed point of the multi-valued mapping $T$ in $X$. 
Notice that

$$
f(x)=d(x, T(x))= \begin{cases}\frac{x}{2}, & \forall x \in[0,1), \\ x-\frac{1}{4}, & \forall x \in[1, \infty)\end{cases}
$$

and

$$
\liminf _{x \rightarrow 1} f(x)=\frac{1}{2}<\frac{3}{4}=f(1)
$$

which implies that $f$ is not lower semi-continuous at 1 . Thus Theorem 3.1 in [2], Theorem 2.1 in [4], Theorem 2.2 in [5] and Theorem 6 in [8] could not be used to judge the existence of fixed points of the multi-valued mapping $T$ in $X$.

\section{Competing interests}

The authors declare that they have no competing interests.

Authors' contributions

All authors read and approved the final manuscript.

\section{Author details}

'Department of Mathematics, Liaoning Normal University, Dalian, Liaoning 116029, People's Republic of China. ${ }^{2}$ Department of Mathematics and RINS, Gyeongsang National University, Jinju, 660-701, Korea. ${ }^{3}$ Department of Mathematics, Gyeongsang National University, Jinju, 660-701, Korea.

\section{Acknowledgements}

The authors would like to thank the referees for useful comments and suggestions. This research was supported by the Science Research Foundation of Educational Department of Liaoning Province (L2012380).

Received: 25 August 2014 Accepted: 4 December 2014 Published: 18 Dec 2014

\section{References}

1. Kada, O, Suzuki, T, Takahashi, W: Nonconvex minimization theorems and fixed point theorems in complete metric spaces. Math. Jpn. 44, 381-391 (1996)

2. Feng, YQ, Liu, SY: Fixed point theorems for multi-valued contractive mappings and multi-valued Caristi type mappings. J. Math. Anal. Appl. 317, 103-112 (2006). doi:10.1016/j.jmaa.2005.12.004

3. Nadler, SB Jr: Multi-valued contraction mappings. Pac. J. Math. 30, 475-488 (1969)

4. Klim, D, Wardowski, D: Fixed point theorems for set-valued contractions in complete metric spaces. J. Math. Anal. Appl. 334, 132-139 (2007). doi:10.1016/j.jmaa.2006.12.012

5. Ćirić, LB: Multi-valued nonlinear contraction mappings. Nonlinear Anal. 71, 2716-2723 (2009). doi:10.1016/..na.2009.01.116

6. Liu, Z, Sun, W, Kang, SM, Ume, JS: On fixed point theorems for multi-valued contractions. Fixed Point Theory Appl. 2010, Article ID 870980 (2010). doi:10.1155/2010/870980

7. Latif, A, Abdou, AAN: Multivalued generalized nonlinear contractive maps and fixed points. Nonlinear Anal. 74, 1436-1444 (2011). doi:10.1016/j.na.2010.10.017

8. Ćirić, LB: Fixed point theorems for multi-valued contractions in complete metric spaces. J. Math. Anal. Appl. 348 499-507 (2008). doi:10.1016/j.jmaa.2008.07.062

9. Latif, A, Abdou, AAN: Fixed points of generalized contractive maps. Fixed Point Theory Appl. 2009, Article ID 487161 (2009). doi:10.1155/2009/487161

10. Liu, Z, Lu, Y, Kang, SM: Fixed point theorems for multi-valued contractions with w-distance. Appl. Math. Comput. 224, 535-552 (2013). doi:10.1016/j.amc.2013.08.061

11. Lin, L, Du, WS: Some equivalent formulations of the generalized Ekeland's variational principle and their applications. Nonlinear Anal. 67, 187-199 (2007). doi:10.1016/j.na.2006.05.006 